\title{
Unidades didácticas: por una enseñanza asistida de la matemática
}

\author{
Jairo José Flores Morales ${ }^{1}$ \\ Winston Joseph Zamora Díaz ${ }^{2}$
}

\section{Resumen}

$\mathrm{E}_{\mathrm{c}}^{\mathrm{n}}$ la labor magisterial, la correcta planificación didáctica debe considerarse uno de los aspectos impresLindibles que garanticen una apropiada enseñanza matemática en los diversos subsistemas educativos. Actualmente, resulta incoherente pensar siquiera que se pueden lograr aprendizajes competenciales en los estudiantes, sin antes haber estructurado el trabajo que se realizará en las diversas sesiones de clase. De ahí, que las unidades didácticas permiten crear un proceso de enseñanza aprendizaje completo, innovador y con acciones pedagógicas que procuran el alcance de las competencias matemáticas en los estudiantes. Si se toman en cuenta los intereses de los estudiantes, sus motivaciones, los contenidos a tratar, las actividades y materiales a implementar, el uso de los recursos tecnológicos existentes, la regulación de aprendizajes, el proceso constante de evaluación, las competencias a desarrollar en los estudiantes y un rol docente que rompa el esquema conformista y tradicional existente, se tendrá como resultado una enseñanza asistida de la matemática, estudiantes que utilizan en su contexto los aprendizajes matemáticos alcanzados y docentes que indagan sistemáticamente la mejor forma de enseñar por medio de la acción y la investigación.

Palabras Clave: Unidades didácticas; planificación; enseñanza-matemática; aprendizajes-competenciales.

\section{Summary}

In the teaching work, it is necessary to consider an accurate didactic planning as one of the essential aspects that can assure an appropriate mathematical teaching in the different educational subsystems. Currently, it is incoherent to even think that learning abilities can be achieve in the students, without having structured the work that will be carried out in the different class sessions. Therefore, the didactic units allow us to create a complete teaching-learning process, innovative and with pedagogical actions that seeks to reach the mathematical skills in students. If we take into account students' interests, their motivations, the contents to be addressed; the activities and materials to be used, the use of the existing technological resources; the learning regulation, the constant evaluation process, the abilities to be developed in students, and a teaching role that breaks the existing conformist and traditional scheme, then we will have as result an assisted teaching of mathematics. This mean that we will have students who use in their own context the mathematical learnings they have achieved and teachers who are systematically investigating the best way to teach through action and research.

Keywords: Didactic units; planning; mathematics teaching; learning-skills.

\footnotetext{
1 Máster en Didáctica de las Matemática, Profesor del Departamento de Ciencias de la Educación y Humanidades de la Universidad Nacional Autónoma de Nicaragua, Managua, correo: jairofmdjmix@yahoo.com

2 Doctor en Educación e Intervencción Social, Profesor del Departamento de Ciencias de la Educación y Humanidades de Universidad Nacional Autónoma de Nicaragua, Managua, correo: winzamora@yahoo.es
} 


\section{Introducción}

$\mathrm{E}_{\mathrm{m}}^{\mathrm{n}}$ forma progresiva, la enseñanza de la matemática ha sufrido cambios radicales dentro del campo educativo. Diversas investigaciones en esta disciplina han constatado que existen una serie de factores que dificultan el proceso de enseñanzaaprendizaje de la misma.

Estos factores giran en torno a dos situaciones: docente-enseñanza y alumno-aprendizaje. Se ha llegado al punto de culpar a los docentes, estudiantes, padres de familia, sistema de evaluación, metodología empleada, en fin, un sin número de culpables que lo único que ocasiona es ahondar la herida sangrante que replica en las expresiones de nuestros estudiantes:

"Ésta asignatura no me gusta", "para qué me van a servir tantos contenidos", "Mejor ni estudio, pues ya estoy reprobado", "salgo mal porque no le entiendo a mi profesor", "me da clase de a chelín y me evalúa de a cinco pesos", "mi maestra no me motiva en clase".

"Estas realidades se pueden comparar con las siguientes, pero desde el punto de vista magisterial: "el estudiante no estudia", "no tengo apoyo del padre de familia", "sale reprobado porque no pone atención en clase", "el sistema de educativo actual fomenta la pereza estudiantil", "antes se enseñaba mejor".

Todo parece indicar que ambos bandos fueran irreconciliables, pero gracias a diversos hallazgos investigativos, se puede afirmar que para cambiar esos esquemas mentales se necesita una ingeniería didáctica que facilite la enseñanza de la Ciencia Matemática. Ciertamente no es tarea fácil, en muchas ocasiones cuando un docente profundiza en un tema al principio solamente encuentra dificultades y caminos cerrados; sin embargo, se debe estar claro, que sin acciones concretas, sin publicar cuestiones que pongan de manifiesto la falta de compromiso docente y del sistema educativo, jamás se podrán mejorar los factores que envuelven negativamente tanto a docentes en la enseñanza , como a los estudiantes en su aprendizaje, y al sistema educativo mismo en la búsqueda de metodologías que propicien aprendizajes relevantes y pertinentes para la vida.

\section{Revisión de la literatura}

Teniendo en cuenta que la didáctica de la Matemática se enfoca en la construcción de modelos teóricos para explicar los distintos aspectos de enseñanza-aprendizaje de ésta en el marco de los sistemas educativos, ésta no se puede desligar de los diversos problemas existentes en el campo de la enseñanza Matemática, sin antes indagar el porqué de esos problemas y cómo darle un tratamiento idóneo. Actualmente, el primordial agente reflexivo de su propio quehacer es el docente, al respecto Godino (2010) expone:

El mundo de la acción práctica es el campo propio del profesor, el cual tiene a su cargo uno o varios grupos de estudiantes a los cuales trata de enseñar matemáticas. El primer objetivo de un profesor es mejorar el aprendizaje de sus alumnos, de modo que estará principalmente interesado en la acción que pueda producir un efecto inmediato sobre su enseñanza.

Desde esta postura, la reflexión crítica en la acción puede afianzar, desarrollar o modificar el conocimiento profesional, para adaptarlo a las múltiples circunstancias concretas, que se dan en el proceso de enseñanza y aprendizaje. Uno de sus propósitos fundamentales es demostrar qué método de enseñanza es más efectivo.

El comprender la naturaleza del pensamiento del estudiante, sus motivaciones, intereses y sobre todo cómo aprende, deberían de ser algunas de las principales tareas del docente antes de iniciar a planificar la enseñanza y por otro lado, usar tales comprensiones para mejorar la educación en Matemática.

Resultaría excelente lograr que los estudiantes adquieran fascinación por la Matemática y que a su vez lo conviertan en estudiantes competentes, tal como lo define la Organización para la Cooperación y el Desarrollo Económico OCDE (2003), al decir que la competencia Matemática es:

La capacidad de un individuo para identificar y entender el papel que las matemáticas tienen en el mundo, hacer juicios bien fundados y usar e implicarse con las matemáticas en aquellos momentos en que se presenten necesidades en la vida de cada 
individuo como ciudadano constructivo, comprometido y reflexivo.

Por ende, se tiene que empezar a dar cambios radicales en nuestra forma de planificar nuestra labor docente. Ya no se puede seguir pensar que la mucha cientificidad que sabe el docente, le resultará sencillo enseñarlo. Acertadamente Díaz (1994), refiere que: "la peor expresión sería afirmar que si uno sabe bien un tema, le es posible enseñarlo; esta expresión es un rechazo cínico a la dimensión teórica de la educación".

Consideramos que, en nuestro país, ha llegado el momento de darle importancia a propuestas didácticas que buscan fortalecer la enseñanza matemática. Todo esto, desde una perspectiva novedosa que busca minimizar los impactos desfavorables que ocasionan las prácticas docentes tradicionalistas y despreocupadas. No existe razón para no tratar vitales temáticas que fomentarían una lucha anuente por una enseñanza y aprendizaje de calidad, aspectos como:

- Fracasos en los intentos por desarrollar una apropiada comprensión de las matemáticas; contenidos desvinculados con el contexto en que vive el estudiante.

- El rol docente, continúa quedándose en el protagonismo tradicional durante el proceso de E-A.

- La falta de sistematización y evaluación de la práctica docente en lo relacionado a la enseñanza de las matemáticas.

- El caso de las pruebas de ingreso a las Universidades como la Universidad Nacional Autónoma de Nicaragua (UNAN) y la Universidad Nacional de Ingeniería (UNI).

- Poca utilización de herramientas tecnológicas en la enseñanza de matemática; disposición al cambio por parte del profesorado.

\section{Materiales y método}

El presente estudio se enmarca en el paradigma cualitativo porque es una actividad sistemática orientada a la comprensión en profundidad de fenómenos educativos y sociales, a la transformación de prácticas y escenarios socioeducativos, a la toma de decisiones y también hacia el descubrimiento y desarrollo de un cuerpo organizado de conocimiento (Bisquerra, 2009). Desde esta perspectiva, Hernández et al. (2010) explican que en el paradigma cualitativo el enfoque o diseño "es el abordaje general que utilizará en el proceso de investigación (p. 492). En este sentido, está investigación tiene un enfoque hermenéutico, porque se interpretaron los significados de textos y acciones en la enseñanza y aprendizajes de las matemáticas.

Por tales razones, se utilizó como método el análisis de contenido, el cual ayudó a describir los documentos en sus partes esenciales. Para ello, se empleó la técnica de revisión de documento, donde nos centramos en los argumentos, razones, propósitos y los significados que los documentos presentan sobre la formación de profesores de matemática. Por consiguiente, se construyó una guía de revisión documental como instrumento, que permitió analizar los libros, artículos científicos, artículos en prensa, leyes, currículo y programas de formación, políticas y normas institucionales.

\section{Resultados y discusión}

De la revisión de la literatura surgen algunas preguntas de cómo docentes de Matemática debemos innovar: ¿Cuál ha sido mi rol ante éstas problemáticas?, ¿Por qué sólo se consiguen aprendizajes en aquellos estudiantes con potencialidades para aprender y en los otros casos, no se logra el aprendizaje aunque el profesor sea competente?, ¿Qué debemos hacer para enfrentar y resolver ésta situación?, ¿cómo planificar la docencia?, y una de las más importantes, ¿Estoy dispuesto a utilizar la didáctica como eje fundamental para lograr una enseñanza asistida de la Matemática?

A cada interrogante se puede responder si nuestra docencia la convertimos en un acto constante de evaluación. Evaluación que serviría para darle un giro a la práctica docente y mejorar la planificación didáctica enfocadas a entender cómo aprenden nuestros estudiantes.

Acertadamente Pérez (2008), plantea la necesidad de analizar las vías fundamentales por las que los seres humanos adquieren significados (aprenden), ya que esto constituye un referente obligado para el desarrollo competente del quehacer docente. Es decir, es necesario tener claro cómo aprende al estudiante, ya que la tarea del profesor será la de apoyar estas formas de aprendizaje. Todo esto es posible mediante una acción que es inherente al rol docente y que todos conocemos como planificación. 
Zabalza (2009), en su libro Competencias Docentes del Profesorado Universitario señala que planificar el proceso de enseñanza-aprendizaje es la competencia con la que debe despuntar quien asume el rol de profesor universitario. De ahí que la planificación es lo que concibe la actuación docente, el tratamiento de los contenidos, la contextualización de los mismos y el curso como un proyecto de desarrollo.

A través de la planificación es posible enfrentarse al control de una serie de variables a las que el profesorado se enfrenta durante el desarrollo de la práctica educativa. Todo lo relacionado con la planificación debe ser concreto a manera de una documentación. Pero además de pensar en los contenidos temáticos a transmitir, debemos tomar en cuenta los siguientes cuestionamientos:

¿Cómo averiguar lo que saben mis estudiantes?, ¿Qué deben aprender hoy mis estudiantes?, ¿Para qué deben aprenderlo?, ¿Con qué medios o recursos puedo lograr esos aprendizajes?, ¿Cómo puedo fomentar/provocar los aprendizajes? ¿Con qué actividades?, ¿Cómo puedo junto con mis estudiantes reconocer su progreso?, ¿Cómo puedo saber si mi actuar docente ha ayudado o perjudicado?, ¿Cómo evaluar realmente? y ¿está bien lo que evalúo?

Es por eso que para conseguir lo antes expresado sobre la práctica educativa es ineludible realizar la planificación del acto educativo. Para ello es necesario apropiarse de una de las tantas formas existentes. Llega el momento de decidir de qué manera o en qué formato quedará mejor reflejado todas las cuestiones que implica esa planificación.

Después de haber realizado diversos trabajos investigativos en didáctica de la matemática, proponemos una planificación por medio de unidades didácticas. Las unidades didácticas son una forma de programación o planificación de la enseñanza en un tiempo determinado, en la misma se pretende incluir al máximo los elementos que intervienen en el proceso educativo.

Podemos decir, que la unidad didáctica es la interrelación de todos los elementos que intervienen en el proceso de enseñanza-aprendizaje con una coherencia interna metodológica y por un período de tiempo determinado. Entre sus principales ventajas tenemos que:
- Puede abarcar el tratamiento de varios contenidos.

- Permite relacionar la teoría con la práctica.

- Orienta el actuar del docente y del estudiante en cada momento.

- Evita el papel monólogo del docente.

- Como alternativa es propio de la metodología constructivista.

- Contiene el qué, el para qué y el cómo del desarrollo de la clase.

- Orienta el proceso de evaluación en todo momento.

Entre sus elementos tenemos la descripción de la unidad didáctica, los objetivos didácticos, contenidos de aprendizaje, secuencia de actividades, recursos materiales, organización del espacio-tiempo y la evaluación. Cada uno de estos elementos cumple con los estándares que actualmente se exigen en los distintos niveles de educación nicaragüense.

Con esta forma de planificar, el docente presta atención a la organización de la enseñanza y el aprendizaje, lo que los estudiantes aprenden y fundamentalmente de cómo se lleva a cabo este aprendizaje (Godino, 2004). Por otro lado, el contexto donde se desarrolla dicho proceso evidencia una ruptura clara de paradigmas que no benefician en lo absoluto a la enseñanza Matemática como tal.

Se hace evidente, que una planificación inadecuada desfavorece la obtención de competencias, más aún, si las actividades que se presentan son una repetición que el estudiante realiza en las actividades ejemplificadas por su maestro, de ahí la importancia de las unidades didáctica y alfabetizar al estudiantado en matemática de una forma diferente. Eficazmente Rico (2006) expresa:

Alfabetización o competencia matemática general se refiere a las capacidades de los estudiantes para analizar, razonar y comunicar eficazmente cuando enuncian, formulan y resuelven problemas matemáticos en una variedad de dominios $\mathrm{y}$ situaciones. Un buen nivel en el desempeño de estas capacidades muestra que un estudiante está matemáticamente alfabetizado o letrado, reducir la noción de alfabetización a sus aspectos instrumentales más básicos, al simple dominio de conceptos y técnicas, puede resultar excesivamente elemental (pp. 276-277). 
Se hace necesaria una planificación innovadora que logre alcanzar dichas competencias. Si nos situamos en nivel de planificación del docente, las componentes en las que se concreta el currículo en cada una de sus dimensiones son los contenidos, los objetivos, la metodología y la evaluación; el modelo de los organizadores del currículo le suministra una serie de herramientas de cara al diseño de actividades de enseñanza y aprendizaje (Lupiáñez, 2009).

En las unidades didácticas, se debe realizar una cuidadosa selección de las tareas y situaciones didácticas que proporcionen oportunidades a los estudiantes para indagar problemas significativos para ellos y sobre todo relevantes desde el punto de vista matemático, formular hipótesis y conjeturas, utilizar diversos tipos de representaciones, realizar buenas preguntas, validar sus soluciones y comunicarlas a otros, dentro de un clima cooperativo y científico, todo esto permite que la interrelación entre los diferentes contenidos sea máxima (Zabala , 2008). Razón fundamental para usar contratos didácticos que proporcionen responsabilidad tanto del docente, como del estudiante.

Los estudiantes aprenden con tareas presentadas en forma de juegos, aprovechando la tendencia natural que tienen a formar grupos, utilizando el aprendizaje cooperativo como estrategia de atención a la diversidad, fomentando la metacognición, e incluso los problemas matemáticos convenientemente escogidos y dosificados, pueden ser muy útiles para el desarrollo del pensamiento matemático, y a la vez, ampliar las estrategias apropiadas en los estudiantes para cada situación conflictiva (Alcina et al,2004). Esta forma de planificación permite, aprender de forma comprensiva, potenciar el explicitación de ideas, conocer las características y habilidades de los estudiantes y valorar los errores de los estudiantes como elemento indispensable para enrumbar los aprendizajes (Dolors \& Montserrat, 2008).

Cabe señalar, que el modelo evaluativo de esta forma de planificación rompe el esquema que lamentablemente aún perdura en nuestros días, al evaluar exclusivamente resultados, obviando todo el proceso. La aplicación de una forma de evaluar que regule los saberes de los estudiantes, permite que las actividades plasmadas en la unidad didáctica sirvan en primera instancia para obtener aprendizajes competenciales, pero a la vez, para autorregular el propio aprendizaje obtenido por el estudiante. Aspectos como los señalados anterior- mente, son los que hacen de las unidades didácticas una propuesta alternativa que apunta hacia una enseñanza asistida de las matemáticas en cualquier nivel escolar.

\section{Conclusiones}

Con el deseo incansable de incitar a la reflexión, y luego de haber discurrido sobre una problemática latente, de largo recorrido, pero de menospreciada atención, cerramos este análisis, estimulando los diálogos conjuntos que orienten propuestas alternativas que puedan abrir camino hacia una inmersión de las matemáticas. A continuación, presentamos a manera de conclusión aspectos que induzcan hacia una reflexión crítica, y que, aunque no son la panacea, consideramos merecen atención.

Tradicionalmente hemos enseñado matemáticas expresándole a nuestros estudiantes que sirven para todo, pero muy pocos o casi ninguno de nosotros (profesores) las hemos utilizado para sobresalir en algún evento científico, para promover la innovación, y a veces ni para resolver acciones cotidianas. Debemos reconocer que las matemáticas seguirán siendo una ciencia integral y un complemento fundamental para el desarrollo de la ciencia y la tecnología, pero eso no lo veremos concretar en nuestro entorno mientras sigamos aprendiendo y enseñando matemáticas sin justificar su historia, el porqué, el cómo aplicarlas y para qué. Estos elementos que predominan con carencia en este proceso son los que permitirían la mencionada motivación.

Debemos evidenciar la necesidad de generar cambios en los procesos educativos de las matemáticas, esto desde un enfoque que fortalezca la contextualización de los contenidos y por ende, la inmersión matemática. Asumir con ahínco que la labor docente frente al desafío de aprender y enseñar matemáticas no es una actividad de abordaje sencillo, por lo que requiere de mucho empeño, de formación permanente y dinámica.

Debe ser una prioridad desde los distintos subsistemas, principalmente en la universidad, promover y desarrollar prácticas didácticas que estimulen la investigación Matemática, la incorporación de las Tecnologías de la Información y Comunicación (TIC) como un elemento que permita experiencias manipulativas, creativas y extrapolables a situaciones del contexto, eludiendo enfrascarse en los planteamientos academicistas bibliográficos que 
niegan concretamente la motivación por aprender matemáticas, y por tanto, la trascendencia de ésta.

Alertar a las universidades encargadas de formar profesores de matemáticas, que éstos requieren una formación que estimule la investigación matemática, y les encamine por una didáctica de las matemáticas que incite hacia formas dinámicas y creativas de planificación. Es necesario ahondar en una planificación y abordaje de las matemáticas que estimule a la comunicación de los aprendizajes, al cálculo, a analizar, a la interpretación, al optimizar, al demostrar, a comparar, a gestionar, en fin a acciones que conlleven a que las matemáticas tengan más sentido fuera del aula de clase.

Fortalecer la visión formadora del profesorado de matemáticas por comprender que es necesario entender la epistemología matemática, pero que es pertinente también comprender y dar respuesta a los distintos ritmos de aprendizajes, a la gestión del conocimiento, a la convivencia en el aula; en fin, todo lo implicado al ambiente escolar como espacio tradicional donde acontece el aprendizaje.

\section{Bibliografía}

Alcina, C. Alcalá, M. Aldana, J. Bishop, A. Carbó, L. Colomer, T. Fernández, A. Ferrero, L. García, A. Giménez, J. Hans, J. Monterde, M. Mora, J. Muñoz, J. Pazos, M. Ramos, N. Recarens, E. y Segarra, L. (2004). Matemáticas re-creativas. Barcelona: Graó;20-23.

Bisquerra, R. (2009). Metodología de la Investigación Educativa. Madrid: La Muralla.

Díaz, A. (1994). Currículo y tecnología educativa. Disponible en: http://goo.gl/xvi64C
Dolors, A. y Montserrat, V. (2008). Juegos de expresión oral y escrita. Barcelona: Graó; 5-6.

Godino, J. (2004). Didáctica de las matemáticas para maestros. Granada: Gami,91-95;425-456.

Godino, J. (2010). Perspectiva de la didáctica de las matemáticas como disciplina tecno-científica. Granada: Universidad de Granada.

Hernández, R., Fernández, C., \& Baptista, M. (2010). Metodología de la Investigación. México: McGra-Hill.

Lupiáñez, J. (2009). Expectativas de aprendizaje y planificación curricular en un programa de formación inicial de profesores de matemáticas de secundaria. Obtenida el 14 de febrero de 2013, de http://goo.gl/q6gMh

OCDE. (2003). El marco de evaluación de PISA 2003; Matemáticas, lectura y resolución de problemas del conocimientos y habilidades. Paris. OCDE

Pérez, A. (2008). ¿Competencias o pensamiento práctico? La construcción de los significados de representación y de acción. En: J. Gimeno-Sacristán (Comp.). Educar por competencias, ¿qué hay de Nuevo? Madrid: Morata, pp. 59-102.

Rico, L. (2006). Marco teórico de evaluación en PISA sobre matemática y resolución de problemas. [Versión electrónica].Revista de educación. (Número extraordinario 2006), 275-294.

Zabala, A. (2008). La práctica educativa. Cómo enseñar. Barcelona: Graó; 35-47,179-181.

Zabalza, A. (2009). Competencias docentes del profesorado universitario, calidad y desarrollo professional. Madrid: NARCEA. 